\title{
Pressure Dependence of the Raman Modes Related to the Phase Transitions in Cyclohexane
}

\author{
H. Yurtseven* AND A.M. CEBECI
}

\begin{abstract}
Department of Physics, Middle East Technical University, 06531 Ankara, Turkey
(Received March 15, 2014; revised version January 15, 2015; in final form February 10, 2015)

The pressure dependence of the Raman frequencies for the $\nu_{21}$ mode is studied by using the volume data at room temperature close to the transitions among the phases of III, IV and V in cyclohexane. By determining the pressure dependence of the mode Grüneisen parameter $\gamma_{T}$ in the phases and taking the average values, the Raman frequencies of those modes associated with the phase transitions are predicted through the volume data in cyclohexane. Our predicted Raman frequencies agree with those observed experimentally in this organic molecule. The Raman frequencies of the other modes can be calculated in cyclohexane and also in some molecular crystals using the method given here.
\end{abstract}

DOI: 10.12693 /APhysPolA.127.744

PACS: $63.70 .+\mathrm{h}, 64.70 . \mathrm{Dv}$

\section{Introduction}

Cyclohexane $\left(\mathrm{C}_{6} \mathrm{H}_{12}\right)$ is a component of petroleum and it undergoes various phases under the pressure and temperature conditions. Its phase diagram has been obtained using differential thermal analysis $[1,2]$ and spectroscopic techniques $[3,4]$. As the pressure changes, it exhibits several phases at room temperature as follows:

Liquid-I(cubic)-III(orthorhombic)-IV(monoclinic)-V

Phase II occurs below $186.1 \mathrm{~K}$ as an orientationally ordered structure [4]. Phase $\mathrm{V}$ is considered to have structural similarities with the monoclinic phase IV [5]. The other phases have been obtained up to $10 \mathrm{GPa}$ at room temperature by the Raman spectroscopy [6, 7].

High pressure studies of cyclohexane to $40 \mathrm{GPa}$ using X-ray diffraction have also been reported previously [8]. The phase transitions occur in cyclohexane at $0.5 \mathrm{GPa}$ (I-III), $1.3 \mathrm{GPa}$ (III-IV) and $3.2 \mathrm{GPa}$ (IVV) [7]. This compound shows significant hysteresis effects $[3,6,7]$.

Near the melting point in cyclohexane, it has been observed by neutron scattering that a gradual change in the cross-section occurs [9]. Anomalous behavior of thermal expansivity in some molecular organic compounds, in particular, in cyclohexane occurs at various pressures $(T=299.7 \mathrm{~K})$ near the melting point [10]. It has been suggested that a second-order transformation occurs prior to melting in cyclohexane [10]. Very recently, using the experimental data for the thermal expansion [10], we have established the Pippard relations in cyclohexane near the melting point [11]. In order to investigate the molecular structure of cyclohexane, calculations of molecular volumes have been conducted [12]. Molecular dynamics simulation of liquid-solid phase transition of cyclohexane has been performed [13]. Deuteration $\left(\mathrm{C}_{6} \mathrm{D}_{12}\right)$ effects in phase transitions of cyclohexane have

* corresponding author; e-mail: hamit@metu.edu.tr also been studied [4]. Phase transition and molecular motion of cyclohexane confined in metal-organic framework have been studied by NMR [14].

The vibrational spectra of cyclohexane have been obtained in earlier studies [15-20]. In particular, spectroscopic studies such as Raman [3, 6, 7, 20], infrared $[3,15,20]$, X-ray diffraction $[8,16]$ and neutron powder diffraction [5] have also been reported in the literature to investigate the phase transitions in cyclohexane.

In this study, we correlate between variations of the Raman frequencies and the crystal volume with the pressure (at room temperature) for the phases III, IV and V of cyclohexane by means of the mode Grüneisen parameter. We use the observed Raman frequencies of the $\nu_{21}[7]$ mode and the observed volume data [8] at various pressures for this correlation. The Raman spectra of $\nu_{21}\left(E_{\mathrm{g}}\right)$ of cyclohexane have been obtained previously at various temperatures (mainly phases II and III) [20] and at various pressures (phases I, III, IV, and V) [7]. It was reported that within an interval of $1.5 \mathrm{~K}$ below the phase transition, $\nu_{21}\left(1268 \mathrm{~cm}^{-1}\right)$ band splits into the five components, which confirms the existence of phase III of cyclohexane by the Raman scattering [20]. Very recently, we have calculated the Raman frequencies of the $\nu_{5}$ mode for the phases of I, III, IV, and V in cyclohexane by determining its mode Grüneisen parameter at various pressures [21]. Here, we calculate the Raman frequencies of the $\nu_{21}$ mode with its five components (a, b, c, d and e) at various pressures (room temperature) for the phases III, IV and V of cyclohexane, as observed experimentally [7].

Below, in Sect. 2 we give our calculations and results. In Sect. 3, we discuss our results. Finally, conclusions are given in Sect. 4.

\section{Calculations and results}

The mode Grüneisen parameter can be defined as

$$
\gamma=\frac{\mathrm{d} \ln \nu}{\mathrm{d} \ln V}
$$


which is due to the volume $(V)$ dependence of the frequency $(\nu)$. When the crystal volume and the vibrational frequency depend on the temperature $(T)$ and the pressure $(P)$, the isothermal mode Grüneisen parameter $\gamma_{T}$ can also be defined as:

$$
\gamma_{T}=\frac{V}{\nu} \frac{(\partial \nu / \partial P)_{T}}{(\partial V / \partial P)_{T}}
$$

The pressure dependence of the mode frequency $\nu$ can then be calculated by integrating both sides of Eq. (2) as follows:

$$
\nu_{T}(P)=A(P)+\nu_{1} \exp \left(-\gamma_{T} \ln \left(V_{T}(P) / V_{1}\right)\right),
$$

where the pressure dependent $A(P)$ term can be defined as:

$$
A(P)=a+b P+c P^{2} .
$$

In Eq. (4), the coefficients $a, b$, and $c$ are all constants. In Eq. (3), $\nu_{1}$ and $V_{1}$ represent the frequency and the volume values, respectively, at a given temperature and pressure. The $A(P)$ term has been added to the solution of the frequency $\nu$ from Eq. (2) in order to predict the frequencies at higher pressures.

TABLE I

Values of the coefficients $a_{0}$ and $a_{1}$ according to Eq. (5) for the $\nu_{21}$ mode with its components in the phases indicated for cyclohexane. $\nu_{1}$ values $(P=0)$ of these modes are also given here.

\begin{tabular}{c|c|c|c|c}
\hline \hline Phases & $\begin{array}{c}\text { Raman } \\
\text { mode } \nu_{21}\end{array}$ & $a_{0}\left[\mathrm{~cm}^{-1}\right]$ & $\begin{array}{c}a_{1} \\
{\left[\mathrm{~cm}^{-1} / \mathrm{GPa}\right]}\end{array}$ & $\begin{array}{c}\nu_{21} \\
{\left[\mathrm{~cm}^{-1}\right]}\end{array}$ \\
\hline III, IV, V & $\nu_{21}(\mathrm{a})$ & 1254 & 2.136 & 1254 \\
III & $\nu_{21}(\mathrm{c})$ & 1266 & 5.999 & 1266 \\
IV & $\nu_{21}(\mathrm{~b})$ & 1265 & 1.815 & 1265 \\
IV & $\nu_{21}(\mathrm{c})$ & 1271 & 3.366 & 1271 \\
IV & $\nu_{21}(\mathrm{e})$ & 1275 & 6.22 & 1275
\end{tabular}

TABLE II

Values of the coefficients $b_{0}, b_{1}$ and $b_{2}$ which were determined according to Eq. (6) for the phase V of cyclohexane. The $V_{1}$ value $(P=0)$ is also given here.

\begin{tabular}{c|c|c|c|c}
\hline \hline $\begin{array}{c}V \\
{\left[\AA^{3}\right]}\end{array}$ & $\begin{array}{c}b_{0} \\
{\left[\AA^{3}\right]}\end{array}$ & $\begin{array}{c}b_{1} \\
{\left[\AA^{3} / \mathrm{GPa}\right]}\end{array}$ & $\begin{array}{c}b_{2} \\
{\left[\AA^{3} / \mathrm{GPa}^{2}\right]}\end{array}$ & $\begin{array}{c}V_{1} \\
{\left[\AA^{3}\right]}\end{array}$ \\
\hline Phase V & 119.12 & -1.915 & 0.012 & 119.12
\end{tabular}

The Raman frequencies of the $\nu_{21}$ mode were predicted from the observed volume data [8] at various pressures at room temperature for the phases I, III, IV and V in cyclohexane. For this prediction, we first determined the mode Grüneisen parameter $\gamma_{T}$ of the $\nu_{21}$ mode in phase $\mathrm{V}$. The observed Raman frequencies [7] of this mode at various pressures (at room temperature) were used as the initial data. Therefore, the pressure dependence of the frequency according to the relation

$$
\nu=a_{0}+a_{1} P,
$$

where $a_{0}$ and $a_{1}$ are constants, was fitted to the observed data [7]. Values of the coefficients $a_{0}$ and $a_{1}$ (Eq. (5)) are given in Table I. We then analyzed the observed volume data [8] in the same pressure range according to the relation

$$
V=b_{0}+b_{1} P+b_{2} P^{2},
$$

where $b_{0}, b_{1}$ and $b_{2}$ are constants. By fitting Eq. (6) to the volume data for phase $\mathrm{V}$ of cyclohexane, the coefficients were determined, as given in Table II. By obtaining the pressure dependence of $(\partial \nu / \partial P)_{T}$ and $(\partial V / \partial P)_{T}$ using Eqs. (5) and (6), respectively, we then determined the values of the isothermal mode Grüneisen parameter $\gamma_{T}$ at various pressures (at room temperature) for the $\nu_{21}$ mode with its components (a, b, c, d and e) according to Eq. (2) for the phases of cyclohexane. Variation of the isothermal mode Grüneisen parameter $\gamma_{T}$ with the pressure, which is determined for the component $\nu_{21}$ (a) Raman mode, is given in Table III for the phases III, IV and V of cyclohexane. We also give the pressure dependence of $\gamma_{T}$ for the Raman

\section{TABLE III}

Values of the isothermal mode Grüneisen parameter $\gamma_{T}$ for the Raman mode with its component $\nu_{21}$ (a) at various pressures (at room temperature) in the phases III, IV and V of cyclohexane.

\begin{tabular}{c|c|c|c|c|c|c|c}
\hline \hline $\begin{array}{c}P \\
{[\mathrm{GPa}]} \\
-\gamma_{T}\end{array}$ & 0.76 & 1.21 & 1.33 & 1.85 & 2.43 & 2.82 & 3.21 \\
\hline $\begin{array}{c}P \\
{[\mathrm{GPa}]}\end{array}$ & 3.6 & 4.92 & 5.47 & 5.95 & 6.21 & 8.27 & 9.14 \\
$-\gamma_{T}$ & 0.104 & 0.103 & 0.103 & 0.103 & 0.103 & 0.102 & 0.101
\end{tabular}

TABLE IV

Values of the isothermal mode Grüneisen parameter $\gamma_{T}$ for the $\nu_{21}$ mode with its components at various pressures (at room temperature) in phase $\mathrm{V}$ of cyclohexane.

\begin{tabular}{c|c|c|c|c}
\hline \hline $\begin{array}{c}P \\
{[\mathrm{GPa}]}\end{array}$ & $\begin{array}{c}\nu_{21}(\mathrm{~b}) \\
-\gamma_{T}\end{array}$ & $\begin{array}{c}\nu_{21}(\mathrm{c}) \\
-\gamma_{T}\end{array}$ & $\begin{array}{c}\nu_{21}(\mathrm{~d}) \\
-\gamma_{T}\end{array}$ & $\begin{array}{c}\nu_{21}(\mathrm{e}) \\
-\gamma_{T}\end{array}$ \\
\hline 3.45 & 0.104 & 0.150 & 0.177 & 0.225 \\
3.8 & 0.105 & 0.151 & 0.178 & 0.226 \\
4.56 & 0.103 & 0.149 & 0.175 & 0.223 \\
5.8 & 0.103 & 0.149 & 0.175 & 0.223 \\
5.92 & 0.103 & 0.148 & 0.174 & 0.221 \\
6.57 & 0.103 & 0.148 & 0.175 & 0.222 \\
7.3 & 0.102 & 0.147 & 0.173 & 0.222 \\
8.69 & 0.101 & 0.146 & 0.172 & 0.222
\end{tabular}

TABLE V

Values of the isothermal mode Grüneisen parameter $\gamma_{T}$ for the $\nu_{21}$ (c) mode in phase III (for the pressures from 0.61 to $1.19 \mathrm{GPa}$ ) and the $\gamma_{T}$ values for $\nu_{21}$ (b), $\nu_{21}$ (c) and $\nu_{21}(\mathrm{e})$ in phase IV of cyclohexane.

\begin{tabular}{c|c|c|c|c|c|c|c}
\hline \hline $\begin{array}{c}P \\
{[\mathrm{GPa}]}\end{array}$ & $\begin{array}{c}\nu_{21}(\mathrm{~b}) \\
-\gamma_{T}\end{array}$ & $\begin{array}{c}P \\
{[\mathrm{GPa}]}\end{array}$ & $\begin{array}{c}\nu_{21}(\mathrm{c}) \\
-\gamma_{T}\end{array}$ & $\begin{array}{c}P \\
{[\mathrm{GPa}]}\end{array}$ & $\begin{array}{c}\nu_{21}(\mathrm{c}) \\
-\gamma_{T}\end{array}$ & $\begin{array}{c}P \\
{[\mathrm{GPa}]}\end{array}$ & $\begin{array}{c}\nu_{21}(\mathrm{e}) \\
-\gamma_{T}\end{array}$ \\
\hline 1.35 & 0.0886 & 0.61 & 0.293 & 1.38 & 0.163 & 1.37 & 0.300 \\
1.84 & 0.0885 & 0.77 & 0.293 & 1.86 & 0.163 & 1.85 & 0.299 \\
2.42 & 0.0882 & 0.96 & 0.292 & 2.40 & 0.163 & 2.40 & 0.298 \\
2.84 & 0.0880 & 1.19 & 0.292 & 2.41 & 0.162 & 2.43 & 0.297 \\
- & - & - & - & 2.86 & 0.162 & 2.84 & 0.296
\end{tabular}


mode $\nu_{21}$ with its components in phase $\mathrm{V}$ (Table IV) and in the phases III and IV (Table V) for cyclohexane. We then determined the values of the coefficients $a$, $b$, and $c$ of the $A(P)$ term (Eq. (4)) using the observed Raman frequency [7] and the volume data [8] at the pressures of $3.45,5.92$, and $8.69 \mathrm{GPa}$ for the $\nu_{21}$ mode in the phase V of cyclohexane, as given in Table VI. Similarly, we determined the coefficients $a, b$, and $c$ (Eq. (4)) using the observed Raman frequency [7] and volume data [8] for the $\nu_{21}$ mode with its components in the phases III and IV (also V for $\nu_{21}$ (a)) as given in Table VII.

TABLE VI

Values of the coefficients $a, b$ and $c$ for the $A(P)$ term according to Eq. (4) for the Raman mode $\nu_{21}$ with its components which we determined for the phase $\mathrm{V}$ of cyclohexane. The average values of the mode Grüneisen parameter $\gamma_{T}$ in this phase are also given here (See Table IV).

\begin{tabular}{c|c|c|c|c}
\hline \hline $\begin{array}{c}\text { Raman } \\
\text { mode } \nu_{21}\end{array}$ & $a\left[\mathrm{~cm}^{-1}\right]$ & $b\left[\mathrm{~cm}^{-1} / \mathrm{GPa}\right]$ & $c\left[\mathrm{~cm}^{-1} / \mathrm{GPa}^{2}\right]$ & $-\gamma_{T}$ \\
\hline$\nu_{21}(\mathrm{~b})$ & -0.068 & 5.029 & -1.270 & 0.103 \\
$\nu_{21}(\mathrm{c})$ & -0.112 & 7.433 & -2.296 & 0.148 \\
$\nu_{21}(\mathrm{~d})$ & -0.130 & 8.773 & -3.602 & 0.175 \\
$\nu_{21}(\mathrm{e})$ & -0.136 & 10.81 & -2.884 & 0.222
\end{tabular}

TABLE VII

Values of the coefficients $a, b$ and $c$ for the $A(P)$ term according to Eq. (4) for the Raman mode $\nu_{21}$ with its components which we determined for the phases indicated in cyclohexane. The average values of the mode Grüneisen parameter $\gamma_{T}$ are also given here (See Tables III and V).

\begin{tabular}{c|c|c|c|c|c}
\hline \hline Phases & $\begin{array}{c}\text { Raman } \\
\text { mode } \nu_{21}\end{array}$ & $\begin{array}{c}a \\
{\left[\mathrm{~cm}^{-1}\right]}\end{array}$ & $\begin{array}{c}b \\
{\left[\mathrm{~cm}^{-1} / \mathrm{GPa}\right]}\end{array}$ & $\begin{array}{c}c \\
{\left[\mathrm{~cm}^{-1} / \mathrm{GPa}^{2}\right]}\end{array}$ & $-\gamma_{T}$ \\
\hline III, IV, V & $\nu_{21}(\mathrm{a})$ & 0.123 & 3.027 & 2.288 & 0.104 \\
IV & $\nu_{21}$ (b) & 2.406 & -6.462 & 10.38 & 0.088 \\
III & $\nu_{21}$ (c) & -15.58 & 40.08 & -11.74 & 0.293 \\
IV & $\nu_{21}$ (c) & 1.505 & 0.331 & 6.477 & 0.163 \\
IV & $\nu_{21}$ (e) & 4.072 & -4.742 & 17.43 & 0.298
\end{tabular}

Finally, by knowing the values of $a, b$, and $c$ (Tables VI and VII), the average values of $\gamma_{T}$ and the extrapolated values of $\nu_{1}=a_{0}$ (Table I) with the $V_{1}=b_{0}$ (Table II) at zero pressure (at room temperature), the Raman frequencies of the $\nu_{21}$ mode with its components were calculated as a function of pressure using the volume data [8] according to Eq. (3). Figure 1 gives the pressure dependence of the Raman frequencies of the $\nu_{21}$ mode with the observed data plotted for the phases studied for cyclohexane.

\section{Discussion}

The Raman frequencies of the $\nu_{21}$ mode were calculated using the volume data through the mode Grüneisen parameter for the phases of I, III, IV and V in cyclohexane. As shown in Fig. 1 for the $\nu_{21}$ mode with its components, the Raman frequencies which we calculated using Eq. (3) are in good agreement with the observed ones.

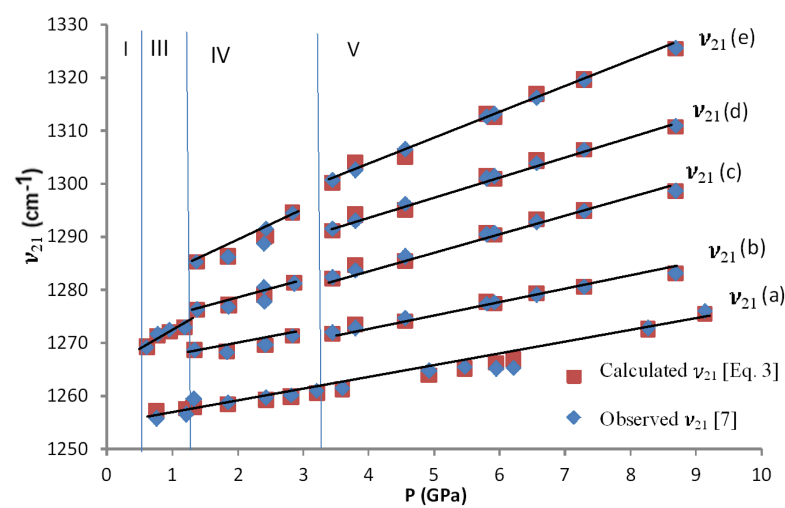

Fig. 1. The Raman frequencies calculated as a function of pressure for the $\nu_{21}$ mode with its components according to Eq. (3) for the phases of III, IV and V of cyclohexane. Observed frequencies [7] are also given here.

To predict the Raman frequencies of the $\nu_{21}$ mode, we used the average values of the mode Grüneisen parameter $\gamma_{T}$ for $\nu_{21}$, as given in Tables VI and VII. The average $\gamma_{T}$ values were used due to the fact that they varied slightly with the pressure within the phases of I, III, IV and V of cyclohexane.

We obtained negative values of the mode Grüneisen parameter $\gamma_{T}$ for the $\nu_{21}$ mode with its components (a), (b), (c), (d) and (e) for the phases studied here. Since the mode Grüneisen parameter $\gamma_{T}$ is essentially the ratio of the frequency shifts to the volume changes (Eq. (2)), as the volume decreases [8] while the Raman frequency $\left(\nu_{21}\right)$ increases [7] with increasing pressure the $\gamma_{T}$ values of this mode become negative.

We also utilized the pressure-dependent term $A(P)$ as a quadratic equation (Eq. (4)) with the coefficients $a, b$, and $c$ which were determined for the Raman mode of $\nu_{21}$ for the phases of III, IV and V in cyclohexane, as given in Tables VI and VII. By taking the average values of the mode Grüneisen parameter $\gamma_{T}$ for the Raman mode of $\nu_{21}$, the pressure-dependent term $A(P)$ was introduced in order to predict the Raman frequencies of those modes at various pressures using the observed volume data [8] for the phases of III, IV and V in cyclohexane.

Regarding the pressure dependence of the Raman frequencies of the $\nu_{5}$ mode, our calculated frequencies [21] show clearly the phase transition between the phases IV and $\mathrm{V}$ as observed experimentally [7]. It has been pointed out that this transition occurs around $3.2 \mathrm{GPa}$ and it is accompanied by a large hysteresis [7]. This transition can also be seen for the component of the Raman mode $\nu_{21}$ (e), as shown in Fig. 1. Other transitions occurring between the phases III and IV and also between the phases I and III for both modes of $\nu_{5}$ [21] and $\nu_{21}$ [7], can be detected by the Raman frequencies shifted as the pressure increases. The Raman spectra have confirmed that the metastable phase occurs at low temperatures at atmospheric pressure and that the stable crystalline phase IV appears above $1.3 \mathrm{GPa}$ at room temperature, 
as pointed out previously [7]. From the Raman spectroscopic measurements up to $40 \mathrm{GPa}$ [6], it has been reported [7] that the stability range of phase IV can extend up to 5 or $5.5 \mathrm{GPa}$ and that a new phase VI can appear between 4.8 and $12.8 \mathrm{GPa}$, which can transform into another phase VII as the pressure is further increased. Using the volume data obtained up to $40 \mathrm{GPa}$, the Raman frequencies of those modes associated with the phase transitions (mainly the $\nu_{5}$ and $\nu_{21}$ Raman modes) can be calculated for those new phases (VI and possibly VII) at higher pressures in cyclohexane. To predict the Raman frequencies of those modes, their mode Grüneisen parameters $\gamma_{T}$ are first determined using the volume data and the Raman frequencies as initial data for those phases. Then, the Raman frequencies can be predicted by the method given in this study. This would then give better structural analysis of all the phases of cyclohexane as one of the organic molecular systems.

\section{Conclusions}

The Raman frequencies of the $\nu_{21}$ mode were calculated as a function of pressure at room temperature using the volume data for the phases of III, IV and V in cyclohexane. For this calculation, the values of the mode Grüneisen parameters $\gamma_{T}$ were determined. As observed experimentally, the Raman frequencies of those modes are shifted to higher values as the pressure varies up to $10 \mathrm{GPa}$, in particular at $3.2 \mathrm{GPa}$, the phase IV changes toward the phase $\mathrm{V}$ in cyclohexane. Our calculated frequencies of the Raman mode $\nu_{21}$ which is associated with the phase transitions in this crystal are in good agreement with the observed data. This indicates that the method of calculating the Raman frequencies from the volume data through the mode Grüneisen parameter in cyclohexane is satisfactory and it can be applied to some other organic molecular crystals.

\section{References}

[1] A. Würflinger, Ber. Bunsenges. Phys. Chem. 79, 1195 (1975).

[2] L. Schulte, A. Würflinger, J. Chem. Thermodyn. 19, 363 (1987).

[3] J. Haines, D.F.R. Gilson, J. Phys. Chem. 93, 7920 (1989).

[4] J. Crain, W.C.K. Poon, A.C. Smith, P.H. Hatton, J. Phys. Chem. 96, 886 (1992).

[5] N.B. Wilding, J. Crain, P.D. Hatton, Acta Crystallogr. B 49, 320 (1993).

[6] M.C. Pravica, Y.R. Shen, M.F. Nicol, Appl. Phys. Lett. 84, 5452 (2004).

[7] V.G. Baonza, Chem. Phys. Lett. 398, 175 (2004).

[8] M. Pravica, Y.R. Shen, Z. Quine, E. Romano, D. Hartnett, J. Phys. Chem. B 111, 4103 (2007).

[9] V.M. Tarina, in: Proc. 4th IAEA Symp. on Neutron Inelastic Scattering Vienna 1968, Ed. C.N. Welsh, Vol. 1, IAEA, Vienna 1968, p. 501.

[10] Ph. Pruzan, D.H. Liebenberg, R.L. Mills, J. Phys. Chem. Solids 47, 949 (1986).

[11] H. Yurtseven, ISRN Thermodynamics 2012, 701209 (2012).

[12] A. Gavezzotti, J. Am. Chem. Soc. 105, 5220 (1983).

[13] A. Brodka, T.W. Zerda, J. Chem. Phys. 97, 5669 (1992).

[14] T. Ueda, K. Kurokava, H. Omichi, K. Miyakubo, T. Eguchi, Chem. Phys. Lett. 443, 293 (2007).

[15] R.J. Obremski, C.W. Brown, E.R. Lippincott, J. Chem. Phys. 49, 185 (1968).

[16] R. Kahn, R. Fourme, D. Andre, M. Renaud, Acta Crystallogr. B 29, 131 (1973).

[17] R.A. Peters, W.J. Walker, A. Weber, J. Raman Spectrosc. 1, 159 (1973).

[18] K.B. Wiberg, A. Shrake, Spectrochim. Acta A 29, 583 (1973).

[19] Y.A. Satatyand, A. Ron, Chem. Phys. Lett. 25, 384 (1974).

[20] U. Rohrer, H.J. Falge, J. Brandmüller, J. Raman Spectrosc. 7, 15 (1978).

[21] H. Yurtseven, B. Bagioglu, J. Mol. Struct. 1067, 61 (2014). 